\title{
Investigation on the hydrodynamic scaling effect of an OWC type wave energy device using experiment and CFD simulation
}

\author{
Saishuai Dai*, Sandy Day, Zhiming Yuan, Haibin Wang \\ University of Strathclyde, Naval Architecture, Ocean and Marine Engineering department, \\ Glasgow, United Kingdom
}

\begin{abstract}
This paper presents a study of the effect of model scale on the performance of a fixed Oscillating Water Column (OWC) type Wave Energy Converter (WEC). Tank tests at two different scales, including the effect of scaling of the test tanks to minimise the bias introduced by different wave blockage effects. CFD simulations based on Reynolds Average Navier Stokes (RANS) method were then carried out for both scaled OWCs to investigate whether CFD simulation is able to reproduce the scale effect. Comparison between the tank test results and the CFD simulation results suggests that CFD simulation is capable of reproducing the hydrodynamic scaling effect with a good accuracy. Results also suggest that the hydrodynamic scaling effect is mainly introduced by the Reynolds number effect for cases investigated in the current study.

Keywords: Tank test, CFD simulation, Scale effect, Wave energy, Oscillating Water Column;
\end{abstract}

\section{Introduction}

Being one of the promising renewable energy technologies, WECs have attracted worldwide attention during the last few decades as one of the more 4 promising marine renewable energy technologies. Detailed reviews of wave

\footnotetext{
* Corresponding author

Email address: saishuai.dai@strath.ac.uk (Saishuai Dai)
} 
5 energy technologies can be found in several studies, for instance, [1, 2] and

6 3. Among all the proposed WEC technologies, the Oscillating Water Column

7 (OWC) type WEC is probably one of the most extensively studied technologies

due to its simple working principle [2].

$9 \quad$ Along with tank test, numerical studies of OWC type WECs have played an 10 important role in accelerating the evolution of OWC technology. For example,

11 Evans 4 derived the theoretical maximum efficiency for a 2D fixed symmetric

12 OWC device by assuming that the OWC surface moves as a weightless rigid

13 piston. Later Sarmento and Falcão 5] improved the theory by allowing OWC surface variations using a surface pressure method and wave flume experiments were carried out to validate the surface pressure theory [6]. With the help of rapid development of computer technology, researchers started to simulate the OWC problem with more advanced methods, such as BEM [7] and CFD [8. Different aspects of the OWC technology have been extensively studied by several researchers, such as hydrodynamic performance [9], optimisation of the OWC geometry [10] and optimisation of turbine-induced damping [1] etc.

Although significant progress on the development and understanding of the OWC technology has been made recently, there are still several challenges to overcome in performance prediction. The effect of model scale is probably one of the critical issues in the early development stage, since the assessment of the full-scale device performance is normally extrapolated from a model scale experiment or simulation result at the early stage. To fill the gaps in theory and guidelines for the requirements of scale testing of a WEC, Sheng et al. [12] presented a theoretical analysis on the scaling of physical modelling and power take-off system. In order to minimise viscous effects, it was recommended that a physical model test shall be carried out with critical Reynolds number above about $10^{5}$. This requirement, however, can not always be fulfilled especially for tests in relatively small wave tanks since the scale (hence Reynolds number) of the test is normally constrained by the tank size. In contrast, numerical simulation methods such as CFD do not have the same limitations of scale. Recently, Elhanafi et al. used an experiment-validated CFD model to investigate the air 
compressibility effect at full scale [13. Although those simulation works are validated against scaled tank test and excellent agreement between the simulation results and experiment results is achieved, there are, however, few published multi-scale tank test data which can validate the capability of simulation tools' to reproduce the aerodynamic and hydrodynamic scale effects.

Recently, Viviano et al. [14] tested a generalized small scale OWC and the results were compared with a similar large scale model to investigate the scale effect. In their study, OWC devices have the same width as the tank width. Therefore, 3D radiation and diffraction effect was excluded. This paper investigates two different scale tank tests of an idealized 3D OWC device. Corresponding CFD simulations are then performed to investigate whether CFD simulation is capable of capturing the hydrodynamic scale effect. This work is structured as below: Section 2 describes the experimental work including a discussion of the uncertainty and error source. Corresponding CFD simulations are described in Section 3 . Section 4 compares the results obtained from the tank tests and CFD. Conclusion and future works are summarized in Section 5

\section{Physical experiments}

Offshore structures (such as offshore platforms) are generally designed in such a way so that the interaction with waves is small. Guidance for these structures on wave blockage may therefore not be well-suited to WECs which are designed to have maximum wave structure interaction. Wave blockage in this context refers to all hydrodynamic effects related to the transverse constraints of the tank walls on the hydrodynamic response - including wave reflection from the tank walls and local variation in flow velocity caused by reduced cross section area due to the presence of the model. Therefore, the impact of wave blockage on results should be carefully considered [15], especially when comparing the performance of two different scale devices, since the effect of wall reflections and flow variations may be confused with the scale effect. 

hereafter.

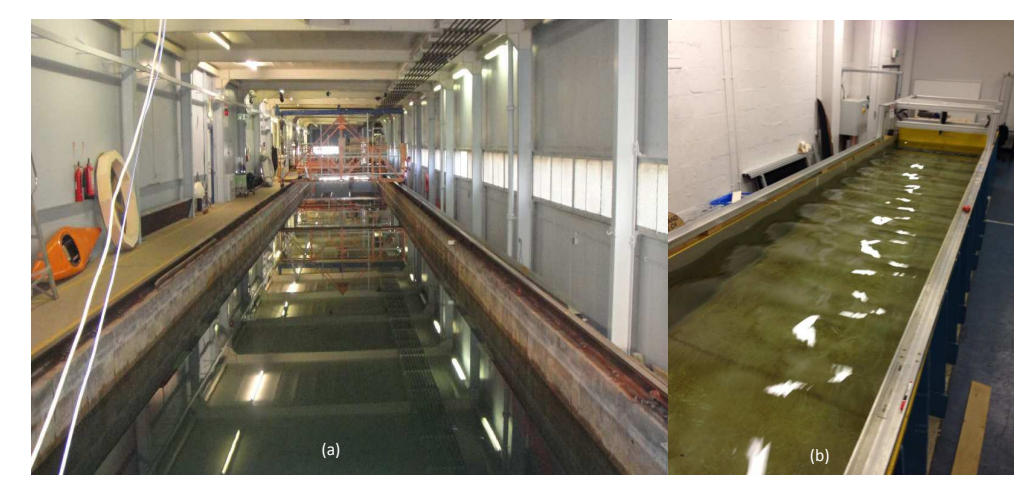

Figure 1: (a) Kelvin Hydrodynamic laboratory. (b) Henry Dyer Hydrodynamic laboratory.

\subsection{Facilities}

In order to minimise the bias from different wave blockage introduced by different tank widths, experiments were carried out in the Kelvin Hydrodynamic laboratory and the Henry Dyer Hydrodynamic Laboratory of the University of Strathclyde as shown in Figure 1. The Kelvin Hydrodynamic Laboratory has a dimension of $76 \mathrm{~m} \times 4.6 \mathrm{~m} \times 2.5 \mathrm{~m}$ with water depth of $2.1 \mathrm{~m}$ and the Henry Dyer Hydrodynamic Laboratory has a dimension of $21.6 \mathrm{~m} \times 1.53 \mathrm{~m} \times 1 \mathrm{~m}$ with water depth set to $0.7 \mathrm{~m}$. Since the cross section dimesion govern wave blockage, it is anticipated that these two tanks will provide similar wave blockage effect when the two models have a scale ratio of $3: 1$. Both tanks are equipped with flap type wave makers and a wave absorbing beach. For convenience, Kelvin tank is denoted as the large tank and Henry Dyer tank is denoted as the small tank

\section{2. $O W C$ device}

Simple acrylic hollow cylinders were selected to model an idealized OWC device for further investigation. Such a simple geometry allows easy scaling of the air compressibility by simply keeping the height of the air chamber the same for both scales [12]. A smooth plastic ring collar was fitted to the bottom of the device in order to have a better control of the sharp corners during the geometry scaling process and at the same time. (see Figure 2 for detail.). The 

information can be found in Table 1 .

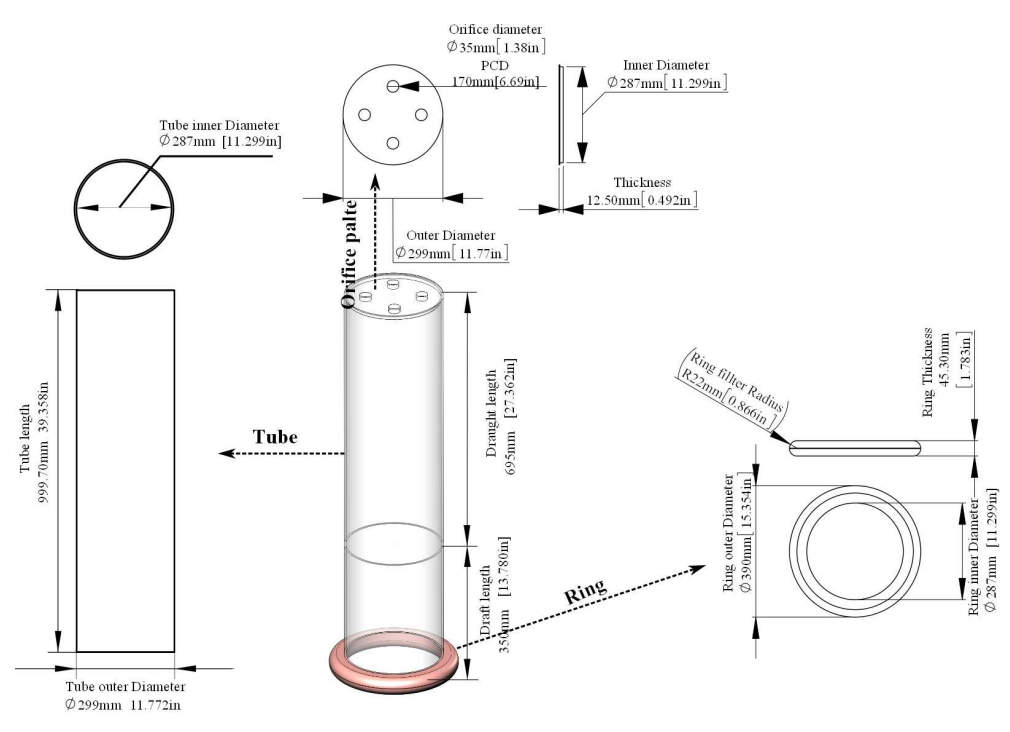

Figure 2: CAD illustration of the large scale device.

Power Take Off (PTO) system was modelled using an orifice plate to simulate an idealised impulse turbine, because it has approximately quadratic pressureflow rate characteristics. This method of modelling the PTO has been used by several researchers, for example, [13] and [16]). Instead of manufacturing several different size orifice plates, 8 equal size and equally spaced circular openings were drilled into the covering lid. By choosing different number of orifices open to the air, different levels of damping could be achieved. More detailed geometry

\section{3. $O W C$ performance and testing procedures}

When assessing the performance of the OWC device, it is critical to assess the available wave power from the incident wave. Conventionally, a reference wave probe is located some distance in front of the device (i.e. between device and wave maker) to measure the incident wave. That measured incident wave information may be different from the wave arriving at the device due to spatial variations of waves in the tank and effects of wave decay. Besides, the wave measured by the reference probe may include the waves due to radiation (from 
Table 1: Geometry details of the two OWC devices, scale ratio 1:3.

\begin{tabular}{llll}
\hline Component & Parameters $(\mathrm{mm})$ & Large scale & Small scale \\
\hline OWC model & total length & 1045.0 & 808.5 \\
& draft & 350.0 & 116.7 \\
Orifice plate & Plate Diameter & 299.0 & 100.0 \\
& Thickness & 12.5 & 4.0 \\
& Orifices Diameter & 35.0 & 11.6 \\
\multirow{3}{*}{ Tube } & Orifices position (PCD) & 170 & 56.5 \\
& Outer Diameter & 299.0 & 100.0 \\
& Inner Diameter & 287.0 & 96.0 \\
& Inner Diameter & 299.5 & 100.5 \\
& Outer Diameter & 390.0 & 130 \\
& Thickness & 45.3 & 15.1 \\
& Fillet radius & 22.0 & 7.5 \\
\hline
\end{tabular}

wave and OWC interaction)and scattering as well as the incident wave. Therefore, in the present work, taking the advantage of the high level of repeatability of the wave makers, the waves were first calibrated at the target location where the devices would be deployed prior to installation of the model. The incident average wave energy flux $\left(P_{\text {avail }}\right)$ can then be determined by the calibrated wave information through

$$
P_{\text {avail }}=\frac{1}{2} \rho g A^{2} C_{g}
$$

where $\rho$ is the density of water, $g$ is the gravitational acceleration, $A$ is the measured wave amplitude and $C_{g}$ is the wave group velocity defined as

$$
C_{g}=\frac{1}{2} \frac{\omega}{k}\left(1+\frac{2 k h}{\sinh (2 k h)}\right)
$$

The $\omega, k, h$ in Equation 2.3 are the circular wave frequency, the wave number and the water depth, respectively. 
Devices were then fixed in the center-line of each tank. Regular waves with non-dimensional frequencies $\left(K h\right.$, here $K=\omega^{2} / g$ ) from 2 to 8 with constant wave height $(0.06 \mathrm{~m}$ for the large scale test and 0.02 for the small scale test.) were then tested. The mean captured power by the OWC device is calculated via

$$
P=\frac{1}{T} \int_{0}^{T} \Delta p(t) q(t) d t
$$

where $T$ is the wave period and $\Delta p(t)$ is the instantaneous pressure difference across the orifice plate. This is measured by a differential pressure transducer installed on the top of the orifice plate. A Honeywell 163PC0D75 $( \pm 622.27 \mathrm{~Pa})$ low pressure differential transducer was used for the large scale tests and a SENSIRION SDP1000-L025 ( $\pm 62 \mathrm{~Pa})$ low differential pressure sensor was employed to measure the pressure for the small scale tests, $q(t)$ in Equation 2.3 is the instantaneous volume flow rate driven by the water column and is defined by

$$
q(t)=A_{w} \frac{\partial \eta}{\partial t}
$$

where $A_{w}$ is the cross section area of the OWC and $\eta$ is the OWC elevation measured by wave probes located in the middle of each device.

To compare the performance of the two devices, the so called capture factor (capture width ratio) is introduced, defined as

$$
C f=\frac{P}{P_{\text {avail }} D_{\text {out }}}
$$

where $D_{\text {out }}$ is the characteristic length of the WEC device. In this case, $D_{\text {out }}$ is the outer diameter of the OWC device (tube).

\subsection{Experiment uncertainty and error}

Uncertainty analysis was performed in line with International Towing Tank recommendation and guidelines $([17,,[18])$ in this paper. The main uncertainty source in the test comes from the instruments used for measurements. This kind of uncertainty (Type B, or systematic uncertainty) can be quantified through 
instrument calibration or stated by the manufacturer. Combined with Type A (random uncertainty) obtained from repeated tests, uncertainty in the physical quantity of interested (e.g. mean captured power) can be calculated using uncertainty propagation analysis. For example, the total uncertainty in the peak mean power captured $(1.1 \mathrm{~W})$ caused by the pressure and volume flow rate measurement is $0.047 \mathrm{~W}$ for the large scale test. Detailed information on uncertainty analysis can be found in the above references. In the present study, the results of uncertainty analysis will be presented via error bars showing $95 \%$ confidence intervals with testing results.

Apart from the uncertainties whose impact can be directly assessed in the form of physical quantities of interest, there exist some uncertainties that cannot be modelled explicitly by uncertainty propagation. For example, the uncertainty in the draft will lead to an uncertainty in the natural frequency of the OWC and hence, in turn uncertainty in the captured power. These kind of uncertainties cannot be directly related to the final power output through uncertainty propagation rules, and therefore can only be quantified separately. The draft was set by visual alignment of the water surface and the draft line; hence the effect of the meniscus may lead to a draft different from the target value. The uncertainty in the draft is estimated to be about 1-2 mm . Similar uncertainties includes the uncertainty in the orifice size measurement, roundness of the OWC tube and the non-horizontality of the water column surface. Although no transversal oscillations were observed during the tests, it should be noted that the non-horizontality of the water column surface may bias the volume flow rate determination since the cross-section area in equation 4 is assumed to be flat and horizontal.

In addition to the uncertainties, there are also some known and unavoidable scaling discrepancies between the two models to available materials and manufacturing accuracy. For instance, the diameter of the tube and the thickness of the orifice plate are not scaled precisely, as shown by Table 1 . 


\section{CFD simulation}

To simulate the air-water two phase interaction problem, a Finite Volume Method (FVM) based software STAR-CCM+ is selected to simulate the two different scale OWC devices. This software has been widely used by several researchers to simulate the OWC problem, for example Lopez [11] and Elhanafi[13].

\subsection{Governing equations and numerical solver settings}

Star-CMM+ uses a predictor-corrector method to link the continuity and momentum equations. The shear stress transport(SST) $k-\omega$ model [19] is selected in current work to model the turbulence. The Volume of Fluid (VOF) [20] method along with high-resolution interface-capturing (HRIC) scheme [21] are employed to resolve the free surface. Simulations are carried out by using a segregated flow model and isothermal ideal gas is selected to account for the air compressibility. The isothermal law is selected in current study because of the fact that the air compressibility and temperature variation at such small scales are negligible. Assuming isothermal avoids solving an ordinary energy transport equation, and hence reduces computational time.

\subsection{Numerical wave tank construction}

Wave generation is realised by specifying the time varying wave particle velocity and wave elevation (hence the phase volume fraction) at the inlet of the CFD domain (Figure 3). Fifth order Stokes wave theory is adopted to calculate the required velocity and wave elevation profile.

Wave damping at the end of the Numerical Wave Tank (NWT) is achieved by introducing a resistance to the vertical motion in the form of a momentum source in a pre-defined zone (for example, zone B in Figure 3] 22. The length of the damping zone B is set to be two wavelength $(\lambda)$ for good absorption performance.

Wave reflection from the inlet boundary is absorbed using the Euler Overlay Method (EOM) 23. This method computes the difference between the analytical wave information and the actual wave information in the specified region 


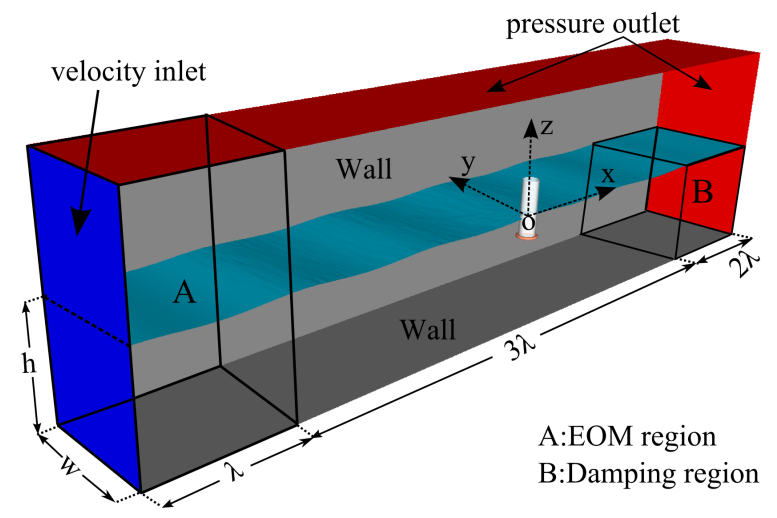

Figure 3: CFD domain and boundary conditions. Here $h$ is the water depth $(2.1 \mathrm{~m}$ for the large scale and $0.7 \mathrm{~m}$ for the small scale.) and $\mathrm{w}$ is half tank width (2.3 $\mathrm{m}$ for the large scale and $0.765 \mathrm{~m}$ for the small scale simulation.)

(zone A in Figure 3, the actual wave is then forced to the analytical wave by adding corresponding source or sink into the governing equations. The source or sink term takes the following form

$$
S(\phi)=-c\left(\phi-\phi^{\star}\right)
$$

Where $S(\phi)$ is the source or sink corresponding to variable $\phi$ (time varying wave particle velocity distribution along the water depth direction and instantaneous wave elevation .). In order to make a smooth transition between the computed and the analytical results, a distance dependent weighting function $c$ is introduced into the source and sink term. The weighting function has the following form

$$
c=c_{0} \cos ^{2}(\pi x / 2)
$$

Where $c_{0}$ is the maximum value of the forcing coefficient and $x$ is the relative distance within the EOM zone ( $x$ equals to zero at the beginning and 1 at the velocity inlet, meaning the forcing takes no effect at the end of the EOM zone and gives the maximum impact at the velocity inlet.). The choice of the value of $c_{0}$ is problem dependent 24], a value of 100 was found to be sufficient and efficient for the present work. 

mesh settings. Those simulations were executed in a pseudo-2D manner which
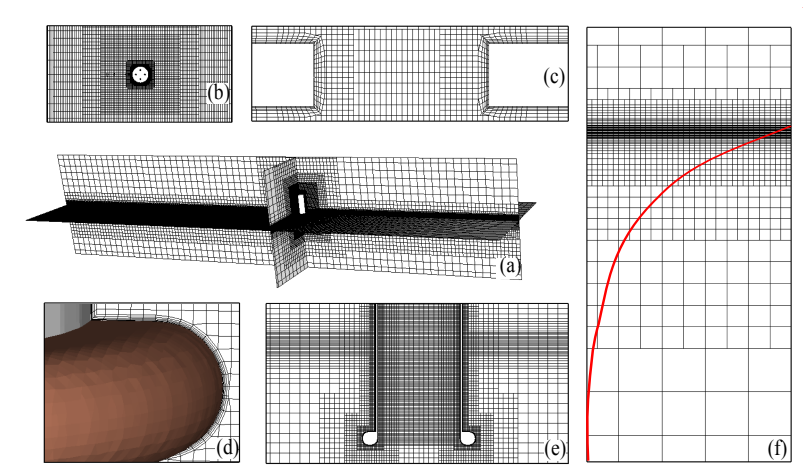

Figure 4: Mesh distribution: (a)overview of mesh distribution, (b) Mesh distribution at the free surface along tank width direction. (c) Mesh distribution around the orifice (sectional view). (d) Mesh distribution around the collar ring. (f) Free surface mesh distribution along water depth direction.

When wave generation is considered, mesh topology normally has a significant impact on the quality of the simulated wave due to numerical dissipation. A denser mesh can normally reduce the numerical dissipation at the cost of longer computation time. The mesh distribution around the free surface was first investigated by performing simulations of a selected wave with different

employed only one cell in the tank width direction. The mesh distribution around the free surface was designed in such a way that the size of the mesh is controlled by the aspect ratio (defined as the ratio between the mesh size in the wave height and the wavelength direction.). The mesh topology in the water depth direction was decided based on the maximum water particle velocity profile. For example, as shown in Figure 4 (f), the mesh gets coarser with increasing water depth as the water particle velocity (illustrated by the red solid line) reduces. Mesh aspect ratio of $1 / 2,1 / 4,1 / 8$ and $1 / 16$ were investigated. the resulting mesh resolution in the wave propagation direction varies from 60 cells in one wavelength to 140 cells in one wave length with a uniform step of 20 cells. The corresponding mesh resolution in the wave height direction is then decided by multiplying the mesh size in wavelength direction with the defined 
aspect ratio. The total number of cells division within the wave height varies from about 5 to about 35 depending on the aspect ratio and the mesh resolution in the wavelength direction.

As for the numerical dissipation caused by the mesh, inappropriate temporal discretization will also lead to dramatic numerical dissipation. A second-order time discretization method is selected to resolve time marching for better accuracy. Time step size $\Delta t$ is decided based on the Courant-Fridrichs-Lewy (CFL) number via

$$
\Delta t=\frac{C F L \cdot \Delta x}{u}
$$

Where $\Delta x$ is the size of a single cell at the free surface in $\mathrm{x}$ direction (see Figure 3 for coordinate system). The denominator $u$ is the wave phase velocity in current study. A CFL number of 0.5 is normally enough to meet the requirement of a second order temporal discretization scheme; in the present study a value of 0.25 is selected to give an extra safe margin.

A regular wave with wave height equal to $0.06 \mathrm{~m}$ at $K h=4.9$ is tested for those proposed mesh settings with correspondingly calculated time step size. It is found that a mesh aspect ratio of $1 / 8$ provides the most economic result for the current study. Figure 5 demonstrates the spatial distribution of the wave elevation along the tank length after 40 seconds simulated physical time. As can be seen, the wave crest height increases with denser meshes in one wavelength indicating the numerical dissipation is relieved with denser mesh. Comparison of the wave height measured at 2 wavelength away from the wave generating inlet and the theoretical wave height suggests the maximum and minimum discrepancy is about $3.7 \%$ and $1.7 \%$, respectively. With an improvement of only $2 \%$, the simulation with 140 cells in the wavelength direction took about 17 hours on a desktop PC with $32 \mathrm{G}$ RAM and 4 core Intel I7-2600 processor $(3.4 \mathrm{GHz})$ while the case with 60 cells only took about 2 hours with the same computer. The 80 cells simulation appears to be the most economic case with a discrepancy of about $2.9 \%$ and 3 hours running time. Therefore, this mesh setting for wave capturing is selected for the OWC simulations. 


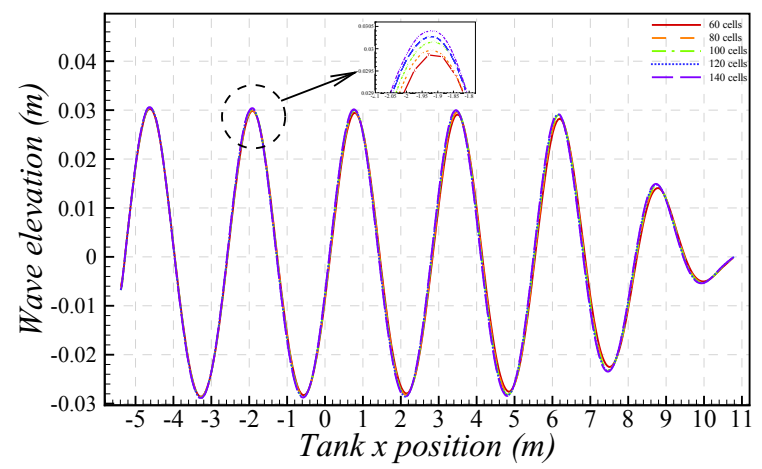

Figure 5: Mesh effect on the free surface elevation spatial distribution along the tank after 40 seconds simulated physical time. The legend states the number of cells in one wavelength.

indicating the validity of the proposed time step size determination method.

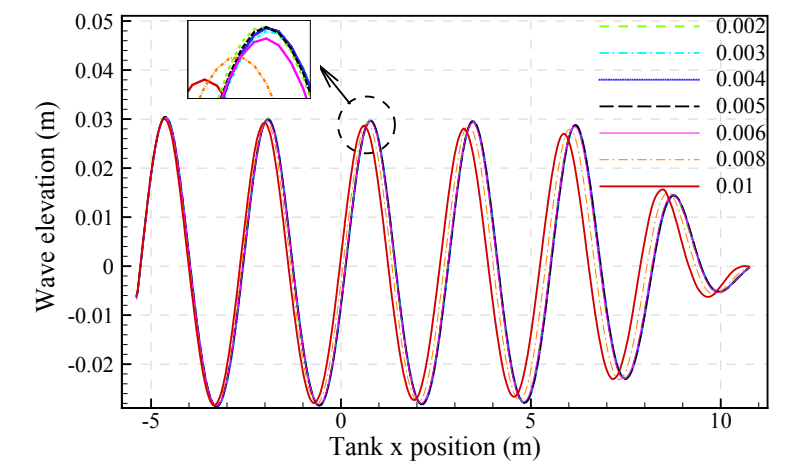

Figure 6: Time step size effect on the wave elevation after 40 seconds physical time simulation.

Simulations using different time step sizes are executed to check the reliability of the proposed time step size determination method. The time step size calculated, based on Equation 2.3 yields about 0.004 seconds. As suggested by Figure6 simulation results converge when the time step size is smaller than 0.005

\section{3. $O W C$ simulation}

The OWC device is then fixed in the middle of the NWT as shown in Figure 3. Boundary conditions are illustrated in Figure 3. It should be noted here that simulations took advantage of the symmetry of the problem about the tank 
centreline; hence, only half of the tank was simulated. The total length of the NWT is set to be $6 \lambda$ which may or may not be sufficient for accurate simulation of the device's performance. This simplification is made due to the large demand of computational resource for the OWC simulations. The dense mesh inside the OWC device (Figure $4(\mathrm{e})$ ), the extra refined mesh around the orifice plate (Figure $4(\mathrm{c})$ ), the mesh refinement outside the OWC device at the free surface and the employment of modelling of the boundary layer at the device surface leads to a mesh with typically 2 million volume cells. The transients of the oscillation of the water column (especially at high frequencies) require long physical time to reach steady state. Along with the required small time step size, this places further demands on the computational resource required. A typical simulation (50 seconds simulated physical time) around the resonant point takes about 64 hours using 48 cores high performance computer (inter Xeon X5650 2.66 GHz CPU).

In the small scale simulation, the small scale OWC device was scaled directly from the large scale simulation according to proper scaling law, rather than reproduced according to the small scale device used in the small scale physical experiment. Hence, the CFD simulation does not have geometry scaling errors. In addition to waves, the mesh distribution and time step size settings for the small scale simulation are directly scaled according to Froude similarity rule as well.

\section{Results}

Study of the OWC device without orifice plate is first carried out to investigate the scale effect on the hydrodynamics without PTO damping. These results are presented in section 4.1. Results of OWC with PTO damping are presented in Section 4.2 .

\subsection{Tests with no PTO damping}

The Response Amplitude Operator, defined as the ratio between the measured OWC motion amplitude and the incident wave amplitude are presented 
in Figure 6 for both tank test and CFD simulations. The RAO obtained from the CFD simulation has been corrected assuming an incident wave amplitude reduction of $3 \%$ due to numerical dissipation based on the NWT study.
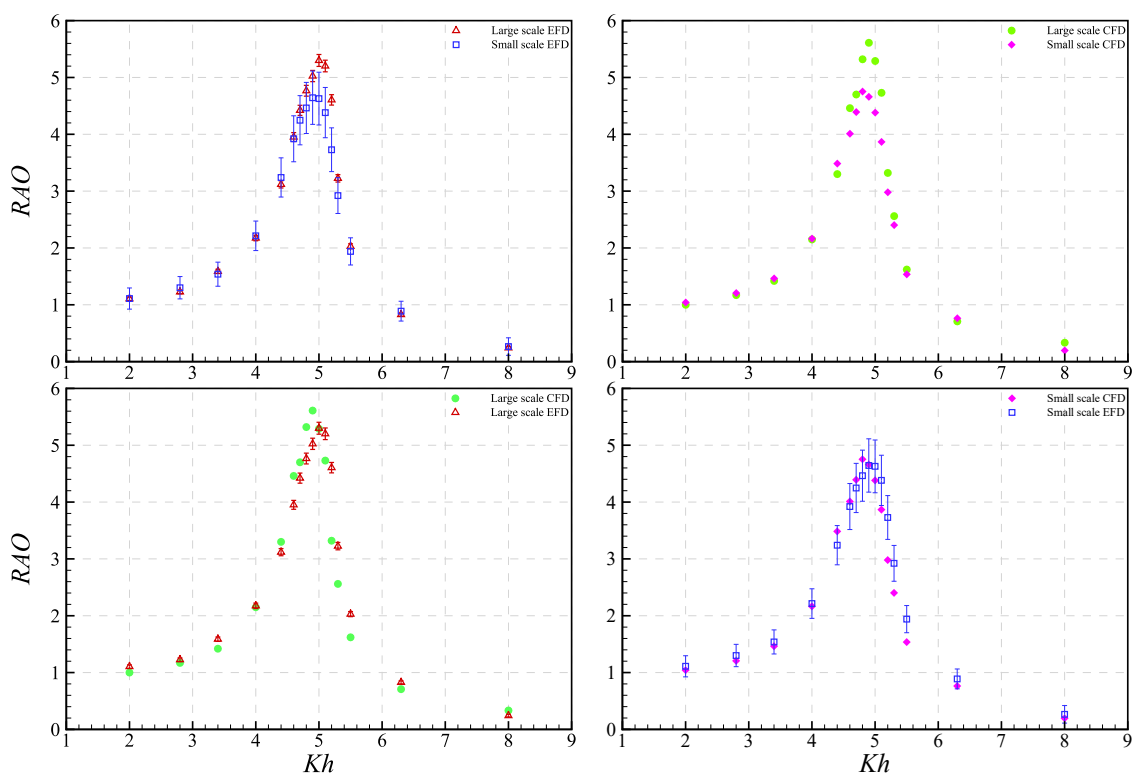

Figure 7: Comparison of RAO obtained from tank test(EFD) and CFD simulation for both large and small scales.

As suggested by Figure 7 the uncertainty in the small scale experiment is much higher than that of the large scale test. This large uncertainty comes from the uncertainty in the wave probes which were used to measure the OWC oscillation and incident wave. The absolute Type B (systematic) uncertainty introduced by those wave probes used in the small scale test is in fact similar to that of the large scale test. However, due to the scaled incident wave amplitude (and hence also the OWC response) the uncertainty in the RAO calculation increased dramatically through uncertainty propagation. In spite of the large uncertainty, it is clear that the response of the small scale test is smaller than that of the large scale test around the peak response frequencies even with such a large uncertainty. The comparison of the large and small scale CFD simulations 
clearly confirmed this observation. The smaller RAO obtained from the small scale model can be explained by the dissimilarity of Reynolds number, that in the small scale OWC is much smaller meaning higher relative viscous losses.

The comparison of the CFD simulation and tank test result suggests the CFD slightly over predicts the peak RAO obtained from tank test for both scales. A frequency shift in the peak response frequency is also observed for both scales. The uncertainty in the draft in the tank test mentioned previously may contribute to the different in natural frequency. This difference in the natural frequency will in turn contribute to the difference in the peak response. Nevertheless, the difference in the peak response period is small. Table 2 lists the peak response period for CFD simulation and tank test.

Table 2: Peak response period obtained by CFD and tank test

\begin{tabular}{lll} 
& Large scale & Small scale \\
\hline CFD & $1.313(\mathrm{~s})$ & $0.758(\mathrm{~s})$ \\
EFD & $1.300(\mathrm{~s})$ & $0.751(\mathrm{~s})$ \\
\hline
\end{tabular}

The maximum and minimum possible peak RAO based on the $95 \%$ uncertainty values for the large and the small scale tests are listed in Table 3 . The corresponding possible maximum and minimum difference in the peak RAO between the large scale and the small scale tests are 1.23 and 0.08 , respectively. The possible relative difference thus varies from $1.5 \%$ to $22.7 \%$ (the minimum relative difference is defined as the ratio of the minimum difference to the large scale minimum possible RAO value, and the maximum relative difference is defined as the ratio of the maximum difference to the large scale maximum possible RAO value.). Calculations based on the measured peak RAO suggest that the small scale test under-predicts the large scale by about $12.4 \%$. Results from the CFD simulation suggest an absolute difference in the peak RAO of 0.86 yielding a relative difference about $15.3 \%$. 
Table 3: Tank test peak RAO and possible minimum and maximum value for the large and small scale OWC.

\begin{tabular}{lllll}
\hline & $\begin{array}{l}\text { Mearsured } \\
\text { peak RAO }\end{array}$ & $\begin{array}{l}\text { Uncertainty } \\
\text { at peak RAO }\end{array}$ & $\begin{array}{l}\text { Maximum } \\
\text { possible RAO }\end{array}$ & $\begin{array}{l}\text { Minimum } \\
\text { possible RAO }\end{array}$ \\
\hline Large scale & 5.300 & 0.105 & 5.405 & 5.195 \\
Small scale & 4.644 & 0.469 & 5.113 & 4.175 \\
\hline
\end{tabular}

\subsection{Tests with PTO damping}

Results are presented here for the case with 4 orifices open to the atmosphere. This configuration gave the maximum power output compared with other conditions (e.g. 8 orifices open to the atmosphere.).

The RAO, the pressure amplitude and the capture factor for OWC devices with modelled PTO damping are presented in Figure 8,Figure 9 and Figure 10 respectively.
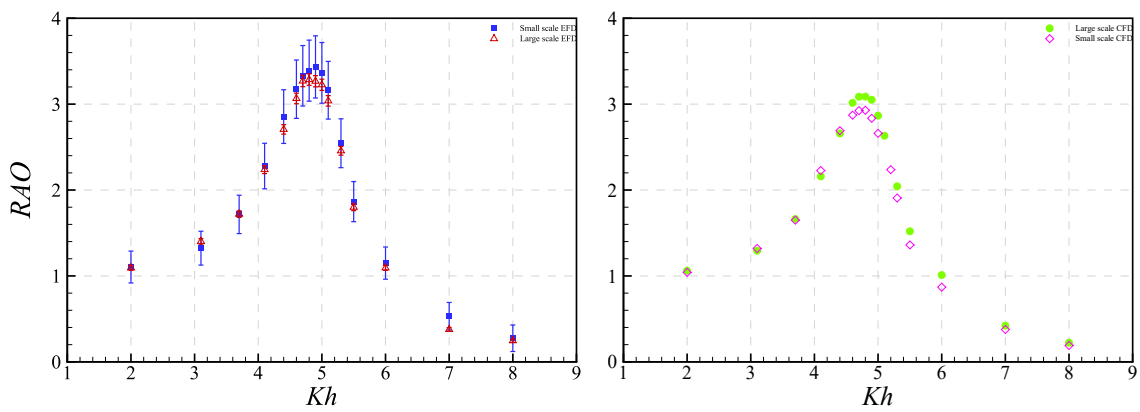

Figure 8: Comparision of RAO obtained from tank test(EFD) and CFD simulation for both large and small scales. PTO damping is set to 4 orifices open to the air.

\section{large and small scales. $\mathrm{PTO}$ damping is set to 4 orifices open to the air.}

The RAO of the small scale tank test suggests a larger response around the peak response frequencies compared with the large scale tank test, however, the large uncertainty covers the large scale results which makes the comparison less reliable. On the other hand, the CFD simulation suggests that the response of the small scale OWC is smaller than that of the large scale OWC. 

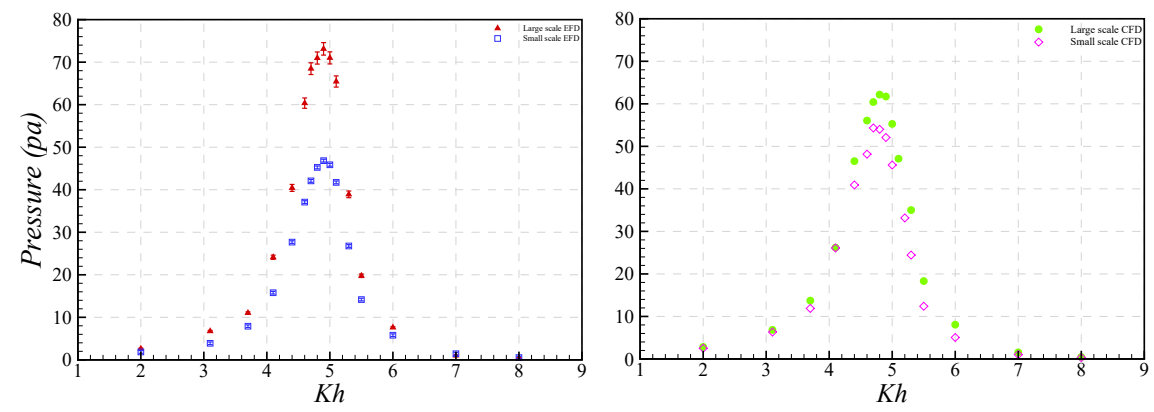

Figure 9: Comparison of pressure amplitude obtained from tank test(EFD) and CFD simulation for both large and small scales. PTO damping is set to 4 orifices open to the air.

The pressure amplitude obtained by the tank test and the CFD simulation both suggest the small scale OWC has a smaller pressure amplitude around the peak response frequency. According to the Froude scaling rule, the pressure amplitude should scale with the geometric scale factor. It can be deduced from Figure 9 that the small scale pressure amplitude is smaller than the pressure amplitude of the large scale after extrapolation to the large scale. Comparing the results between the tank test and the CFD simulation, it can be seen that the peak pressure amplitude of the large scale simulation is smaller than that of the tank test while the small scale simulation has a higher peak pressure amplitude. The smaller pressure amplitude of the large scale simulation can be explained by the NWT dissipation, which results in, the wave amplitude arriving at the OWC device with smaller than the specified wave amplitude. The larger pressure amplitude of the small scale CFD simulation will be explained in section 4.3 .

Both the tank test and the CFD simulation suggest that the small scale capture factor is smaller than that of the large scale. Table 4 lists the measured maximum and minimum possible capture factor for the large and the small scale tank test. Based on the maximum and minimum possible value, the small scale test results under predict the large scale capture factor by about $24.5 \%$ to $37.6 \%$. The calculation based on the measured value suggests that the small 

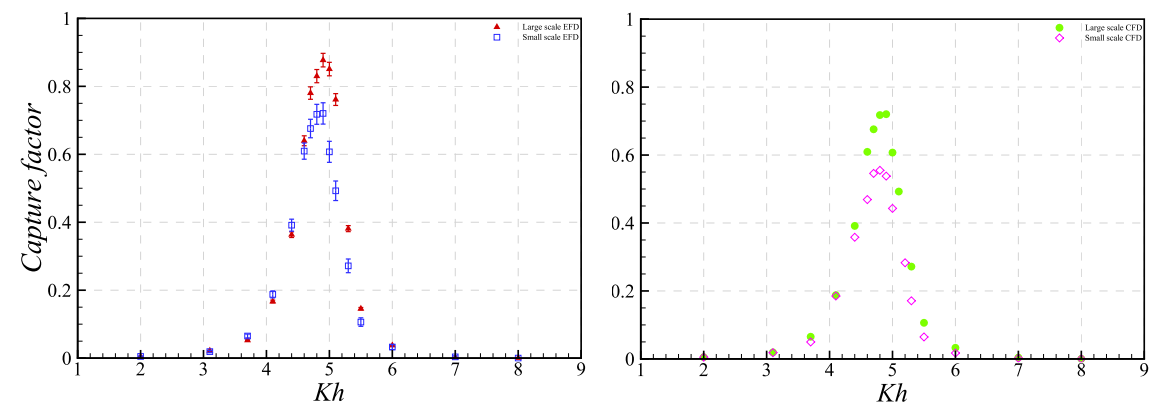

Figure 10: Comparison of capture factor obtained from tank test(EFD) and CFD simulation for both large and small scales. PTO damping is set to 4 orifices open to the air.

scale under predicts the large scale result by about $31 \%$. On the other hand, the CFD simulation results indicate that the small scale simulation under predicts the large scale simulation by about $22.9 \%$.

Table 4: Tank test peak capture factor and possible minimum and maximum value for the large and small scale OWC.

\begin{tabular}{lllll}
\hline & $\begin{array}{l}\text { Mearsured } \\
\text { peak Cf }\end{array}$ & $\begin{array}{l}\text { Uncertainty } \\
\text { at peak Cf }\end{array}$ & $\begin{array}{l}\text { Maximum } \\
\text { possible Cf }\end{array}$ & $\begin{array}{l}\text { Minimum } \\
\text { possible Cf }\end{array}$ \\
\hline Large scale & 0.877 & 0.039 & 0.916 & 0.839 \\
Small scale & 0.602 & 0.031 & 0.633 & 0.571 \\
\hline
\end{tabular}

\subsection{PTO scaling}

The modelled PTO system: the orifice plate, as mentioned previously, has a quadratic pressure-flow rate characteristic as shown by Figure 11 and can be described by

$$
p=\Lambda q^{2}
$$

the damping coefficient $\Lambda$ is a real number describing the relationship between the pressure and the volume flow rate.

As indicated by Figure 11, apart from the small scale tank test, all the other pressure and volume flow rate amplitude relationship are very close to each 


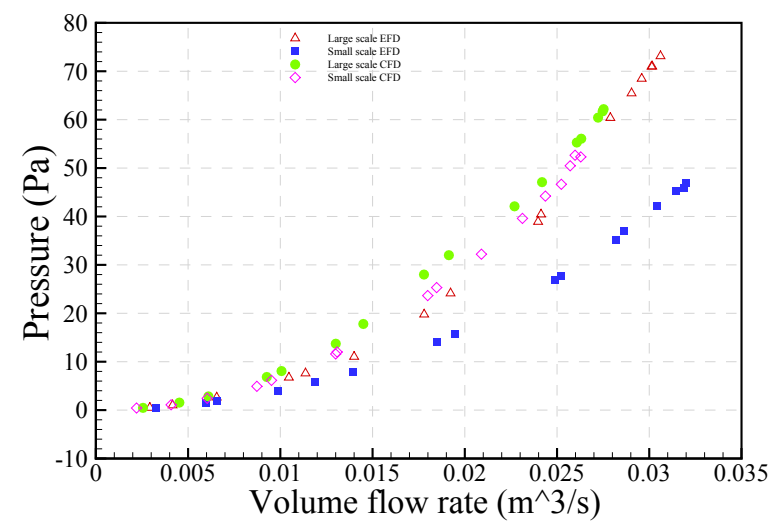

Figure 11: Pressure amplitude and volume flow rate amplitude relationship, here the small scale result are extrapolated to the large scale according to the Froude scaling rule. Each data point corresponding to a single frequency simulation/tank test.

other. This suggests that the damping applied in the small scale tank test is different from the damping used in the CFD simulation.

Following the Froude scaling rule, $\Lambda$ should scale with $s^{-4}$, yielding $1 / 81$ in the present work, here $s$ is the scale factor defined as the ratio between the geometry dimension of the large scale and the geometry dimension of the small scale. The scale factor is 3 in the present study. Figure 12 compared the damping ratio (defined as $\Lambda_{\text {small }} / \Lambda_{\text {large }}$ ) for the tank test and the CFD simulation. This explains why the small scale CFD simulation has a larger pressure amplitude than the small scale tank test.

It is clear that the damping ratio of the tank test is far from the Froudescaled value. This is because the small scale PTO was not scaled correctly due to the errors and uncertainties. The uncertainty in the damping ratio for the tank test is enormous due to the large uncertainty in the small scale OWC elevation measurement. On the other hand, the damping ratio of the CFD simulation is close to the theory ratio compared with the tank test result. However, it is still smaller than the theoretical value.

Figure 13 illustrates the relationship between the damping coefficient $\Lambda$ and the orifice Reynolds number for the large and small scale CFD simulation. The 


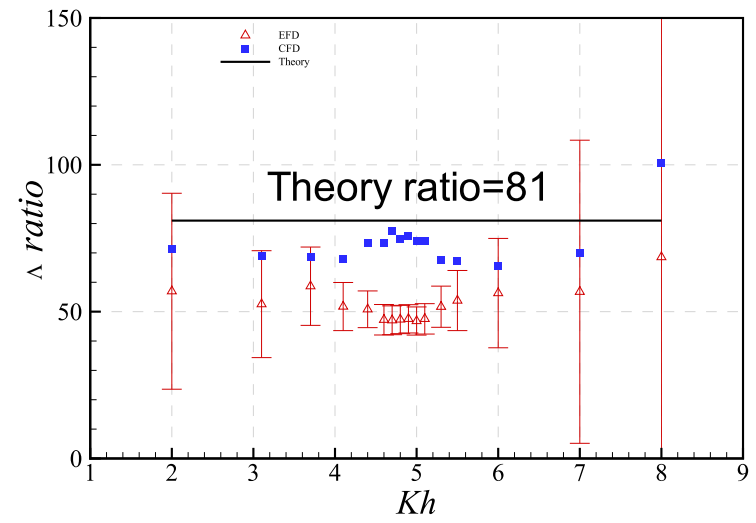

Figure 12: Comparison of the $\Lambda$ ratio between the tank test and the CFD simulation.

orifice Reynolds number is defined as

$$
R e=\frac{D \cdot U}{\nu_{\text {air }}}
$$

where $\mathrm{D}$ is the characteristic length of the orifice plate and is defined as 2 times the orifice diameter (This characteristic length is decided to be the diameter of an orifice whose area is equivalent to the total area of the four orifice), $U$ is the mean air velocity through the orifices, calculated by dividing the OWC volume flow rate by the total orifice area and $\nu$ is the dynamic viscosity of air.

Figure 13 suggests that for the large scale, the damping coefficient $\Lambda$ is smaller at low Reynolds number and increases with increasing Reynolds number. With further increased Reynolds number (up to about 6.5E5), $\Lambda$ is found to reduce to some extent and tends to stabilize. The small scale simulation has a much smaller Reynolds number and it seems like that the extrapolated small scale $\Lambda$ falls into the low Reynolds number region of the large scale $\Lambda$, suggesting the small scale $\Lambda$ experienced Reynolds number effect.

\subsection{CFD simulation of a larger scale $O W C$}

Keeping the air chamber of the OWC device the same height as previous two scale simulations, a further extrapolation of three times larger than the large scale simulation is carried out. Figure 14 shows the comparison of the mean 


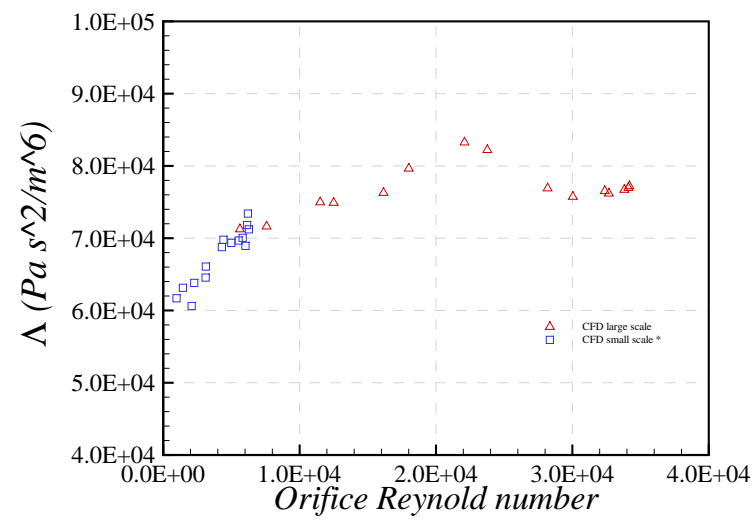

Figure 13: Reynolds effect on the $\Lambda$. Here the $\Lambda$ of the small scale simulation is extrapolated to the full scale according to the Froude scaling rule while the Reynolds number is kept at small scale.

captured power for the three different scales and Table 5 summarises the results and relative differences.

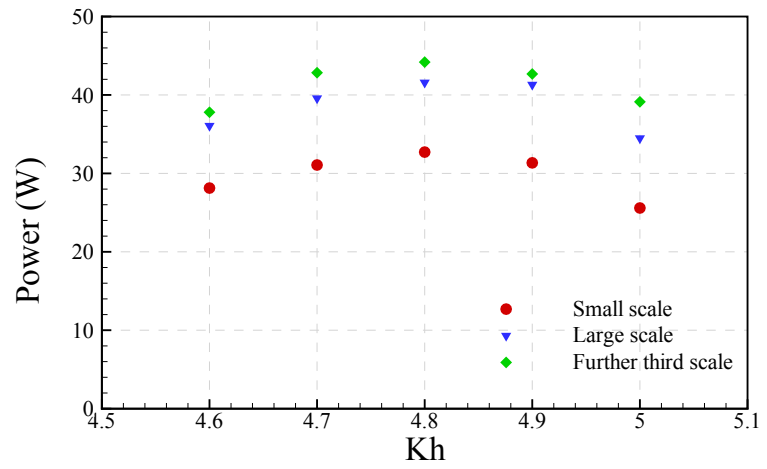

Figure 14: Comparison of the mean captured power for different scales. Power of the small scale and the large scale are extrapolated to the further third scale according to Froude scaling rule.

The large scale simulation under-predicts the values obtained for the further three-times scale by an average of $7 \%$ and the small scale simulation underpredicts the further third scale by an average of $28 \%$. Figure 15 plots the peak mean power captured against the Reynolds number. Here the Reynolds number 
Table 5: Mean power captured for the three different scales. Here the large scale and small scale power are extrapolated to the further 3 times larger scale using Froude scaling rule. The relative difference are calculated based on the further 3 times lager scale.

\begin{tabular}{llllll}
\hline Kh & 4.6 & 4.7 & 4.8 & 4.9 & 5.0 \\
\hline Further 3 times larger scale (W) & 37.80 & 42.84 & 44.19 & 42.68 & 39.13 \\
large scale (W) & 36.06 & 39.57 & 41.58 & 41.31 & 34.48 \\
small scale (W) & 28.13 & 31.07 & 32.72 & 31.35 & 25.59 \\
Relative difference (large scale) & $-5 \%$ & $-8 \%$ & $-6 \%$ & $-3 \%$ & $-12 \%$ \\
Relative difference (small scale) & $-26 \%$ & $-27 \%$ & $-26 \%$ & $-27 \%$ & $-35 \%$ \\
\hline
\end{tabular}

413 is defined according to [12] as

$$
R e=\frac{A \omega D_{o u t}}{\nu_{w a t e r}}
$$

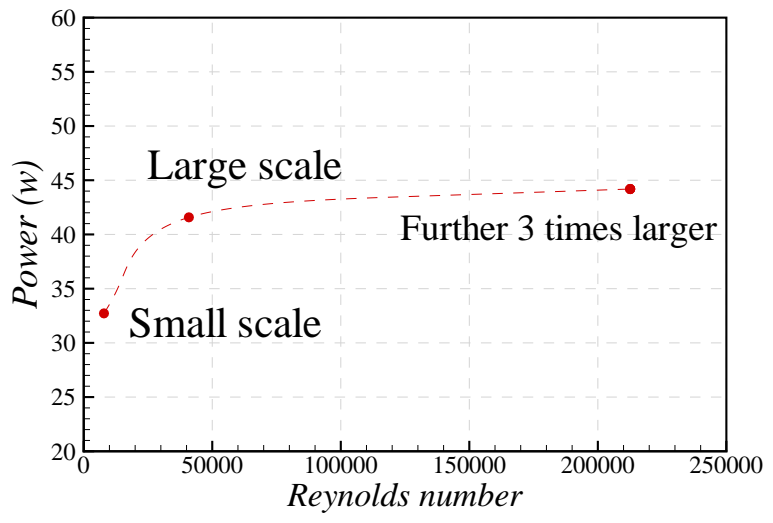

Figure 15: Peak mean captured power against Reynolds number. 


\section{Discussion and conclusion}

This paper presents tank test and CFD simulation of two different scale OWC type WEC. A CFD simulation three times larger again is presented for further investigation of the scale effect.

The comparison between the tank test and CFD simulation of the cases without the modelled PTO suggest that, in spite of the uncertainty in the draft of tank test, the CFD simulation can predict the scale effect quite accurately. The small scale CFD simulation under-predicts the large scale peak RAO by about $15.3 \%$ while the tank tank test suggests a $12.4 \%$ difference.

When the modelled PTO is considered, very precise scaling of the orifice at such scales is difficult for tank test. The damping provided by the orifice is extremely sensitive to the size of the orifice at the small scale tank test. With only $0.07 \mathrm{~mm}$ difference in the orifice diameter (hence $0.28 \mathrm{~mm}$ difference in total since 4 orifices are used.) and $0.167 \mathrm{~mm}$ difference in the orifice plate thickness, the volume flow rate and pressure relationship changed significantly as suggested by Figure 11. In contrast, the CFD simulation is not restricted by these practical scaling issues. The small scale CFD simulation provides a similar volume flow rate and pressure relationship as the large scale simulation. The CFD results indicate that the small scale simulation underestimated the large scale peak capture factor by about $22.9 \%$ while the tank test suggests the small scale tank test under-predicts the large scale by between $24.5 \%$ to $37.5 \%$ considering the uncertainty. The relative difference between the small scale tank test and the large scale tank test without considering the uncertainty is about $31 \%$. The difference between the large scale and the small scale tank test is anticipated to be smaller than $31 \%$ if the orifice of the small scale tank test were able to be perfectly scaled.

The discrepancy between the large scale tank test of the OWC with PTO and the CFD simulation is mainly due to the dissipation introduced by the NWT. CFD simulations have uncertainties introduced by the mesh, the choice of time step etc. A careful study of mesh and time step size impact should be carried 
out to examine the errors and uncertainties for accurate simulation of the OWC device. However, in this study, same numerical settings and mesh strategies are adopted for all those different scales, it is assumed to be reasonable to assume that the errors and uncertainties introduced are unidirectional and have similar relative effect on the final output. Therefore, it will probably not affect the comparison between different scales significantly. In order to accurately simulate the performance of a WEC, it is suggested to calibrate the NWT in advance and adjust the input wave in such a way so that the wave arriving at the device is equal to the required value.

The performance of the modelled PTO system (the orifice plate) is affected by the orifice Reynolds number as indicated by Figure 13 . The orifice Reynolds number is dependent on the motion of the OWC which is not known in advance. Therefore, it is recommended to check the Reynolds number effect on the damping coefficient $\Lambda$ afterwards to check whether the performance of the orifice is strongly affected by the Reynolds number. If so, the orifice Reynolds number should be reported along with the final result.

A further three-times scaled up CFD simulation result indicates that the large scale simulation used here is not affected by the Reynolds number significantly. Judging by the trend line (Figure 15), Sheng's 12] recommendation of critical Reynolds number seems to be a good choice. It should be noted here that for the further three-times scaled CFD simulation, the results may be more affected by the air compressibility which would need further investigation. For example, perform CFD simulation with a more realistic compressible air model such as real gas model.

CFD simulations of the three different scale OWC in current study required similar amount of computation resource since mesh and numerical settings were scaled accordingly. On the other hand, the cost of the tank test increased with the scale (mainly introduced by the cost of the facilities and model.). However, the cost of the small scale tank test is in fact lower than that of the small scale CFD simulations. It is still not cost-effective to investigate an OWC type WEC at small scale (about 1:100 scale of a full scale device) using CFD simulation at 
current stage.

\section{Future work}

Although the effect of side wall reflection is not reported in this work, primary tank test results indicate that a model breadth to the tank width ratio of 0.2 is not enough to ignore the tank width effect for current OWC device. Detailed work on the tank width effect will be reported in the future.

\section{Acknowledgement}

The CFD simulation results were obtained using the EPSRC funded ARCHIEWeST High Performance Computer (www.archie-west.ac.uk). EPSRC Grant no. EP/K000586/1.

Authors would like to thank all reviewers for their valuable time and valuable comments.

\section{References}

[1] J. Cruz, Ocean wave energy: current status and future prespectives, Springer,Berlin. Science \& Business Media, 2007.

[2] A. F. d. O. Falcão, Wave energy utilization: A review of the technologies, Renewable and Sustainable Energy Reviews 14 (3) (2010) 899-918. doi: https://doi.org/10.1016/j.rser.2009.11.003

[3] N. Khan, A. Kalair, N. Abas, A. Haider, Review of ocean tidal, wave and thermal energy technologies, Renewable and Sustainable Energy Reviews 72 (2017) 590-604. doi:https://doi.org/10.1016/j.rser.2017. 01.079 .

[4] D. Evans, A theory for wave-power absorption by oscillating bodies, Journal of Fluid Mechanics 77 (1) (1976) 1-25. 
[5] A. J. Sarmento, A. d. O. Falcão, Wave generation by an oscillating surfacepressure and its application in wave-energy extraction, Journal of Fluid Mechanics 150 (1985) 467-485.

[6] A. Sarmento, Wave flume experiments on two-dimensional oscillating water column wave energy devices, Experiments in fluids 12 (4-5) (1992) 286-292.

[7] Y. M. C. Delauré, A. Lewis, 3d hydrodynamic modelling of fixed oscillating water column wave power plant by a boundary element methods, Ocean Engineering 30 (3) (2003) 309-330. doi:https://doi.org/10. 1016/S0029-8018(02) 00032-X.

[8] Y. Zhang, Q.-P. Zou, D. Greaves, Air-water two-phase flow modelling of hydrodynamic performance of an oscillating water column device, Renewable Energy 41 (2012) 159-170. doi:https://doi.org/10.1016/j.renene. 2011.10 .011

[9] A. Kamath, H. Bihs, i. A. Arntsen, Numerical investigations of the hydrodynamics of an oscillating water column device, Ocean Engineering 102 (2015) 40-50. doi : https://doi.org/10.1016/j.oceaneng. 2015.04 . 043.

[10] F. Mahnamfar, A. Altunkaynak, Comparison of numerical and experimental analyses for optimizing the geometry of owc systems, Ocean Engineering 130 (2017) 10-24. doi:https://doi.org/10.1016/j.oceaneng.2016.11. 054 .

[11] I. López, B. Pereiras, F. Castro, G. Iglesias, Optimisation of turbineinduced damping for an owc wave energy converter using a rans-vof numerical model, Applied Energy 127 (2014) 105-114. doi:https://doi.org/ 10.1016/j. apenergy.2014.04.020

[12] W. Sheng, R. Alcorn, T. Lewis, Physical modelling of wave energy converters, Ocean Engineering 84 (2014) 29-36. doi:https://doi.org/10.1016/ j.oceaneng.2014.03.019. 
[13] A. Elhanafi, G. Macfarlane, A. Fleming, Z. Leong, Scaling and air compressibility effects on a three-dimensional offshore stationary owc wave energy converter, Applied Energy 189 (2017) 1-20. doi:https://doi.org/ $10.1016 / \mathrm{j}$.apenergy.2016.11.095

[14] A. Viviano, S. Naty, E. Foti, Scale effects in physical modelling of a generalized owc, Ocean Engineering 162 (2018) 248-258.

[15] A. H. Day, A. Babarit, A. Fontaine, Y. P. He, M. Kraskowski, M. Murai, I. Penesis, F. Salvatore, H. K. Shin, Hydrodynamic modelling of marine renewable energy devices: A state of the art review, Ocean Engineering 108 (2015) 46-69. doi:https://doi.org/10.1016/j.oceaneng.2015.05. 036.

[16] D.-Z. Ning, R.-Q. Wang, Q.-P. Zou, B. Teng, An experimental investigation of hydrodynamics of a fixed owc wave energy converter, Applied Energy

1) 168 (2016) 636-648. doi:https://doi.org/10.1016/j.apenergy.2016. 01.107 .

[17] ITTC, Procedures and guidelines: Guide to the expression of uncertainty in experimental hydrodynamics, Report (2008).

[18] ITTC, Recommended procedures and guidelines: Uncertainty analysis instrument calibration, Report 7.5-01-03-01 (2008).

[19] F. R. Menter, Improved two-equation k-omega turbulence models for aerodynamic flows, NASA STI/Recon Technical Report N 93 (1992) 22809.

[20] C. W. Hirt, B. D. Nichols, Volume of fluid (vof) method for the dynamics of free boundaries, Journal of computational physics 39 (1) (1981) 201-225.

[21] T. Wacławczyk, T. Koronowicz, Comparison of cicsam and hric highresolution schemes for interface capturing, Journal of theoretical and applied mechanics 46 (2) (2008) 325-345. 
[22] J. Choi, S. B. Yoon, Numerical simulations using momentum source wave-maker applied to rans equation model, Coastal Engineering 56 (10) (2009) 1043 - 1060. doi:https://doi.org/10.1016/j.coastaleng. 2009.06 .009

URL http://www.sciencedirect.com/science/article/pii/ S0378383909000970

[23] J. Kim, J. O'Sullivan, A. Read, Ringing analysis of a vertical cylinder by euler overlay method, in: ASME 2012 31st International Conference on Ocean, Offshore and Arctic Engineering, American Society of Mechanical Engineers, 2012, pp. 855-866.

[24] S. Enger, M. Perić, H. Monteiro, Coupling of 3d numerical solution method based on navier-stokes equations with solutions based on simpler theories (2014). 